\title{
EDUCACIÓN FÍSICA: UNA ASIGNATURA PARA MEJORAR EL RENDIMIENTO ACADÉMICO, LA COGNICIÓN Y LOS VALORES
}

Physical education: a subject to improve the academic performance, the cognition and the values

Educação física: uma matéria para melhorar o rendimento acadêmico, a cognição e os valores

\section{Diego Bernal Rubio (1) \\ M José Daniel Huerta (2)}

(1) Universidad de Salamanca, España. Correo electrónico: diegobernal_@hotmail.com

(2) Universidad de Salamanca, España. Colegio Esclavas del Sagrado Corazón de Jesús. Fundación Educativa ACI. Telf.: +34 923294630 (Ext: 3362). Correo electrónico: marijo@usal.es

\section{Resumen}

Varias investigaciones muestran que la práctica de actividad físico-deportiva aporta múltiples beneficios a nivel cognitivo, motor, psicológico y social. Todas estas ganancias revierten positivamente en los niños durante su escolarización, además de consolidar valores mediante la actividad físico-deportiva en el área de Educación Física. Por este motivo, entendemos que hay que facilitar el ejercicio corporal desde las primeras etapas de la vida, por tratarse del momento clave para fraguar hábitos saludables que acompañarán a las personas a lo largo de su vida.

Después de advertir mejores resultados académicos en países y escuelas donde dedican más horas lectivas a la asignatura de Educación Física, pensamos que a la hora de realizar las reformas educativas estos datos no pueden obviarse si lo que se pretende es la mejora de la calidad educativa.

Palabras clave: actividad físico-deportiva, beneficios, etapa escolar.

\begin{abstract}
Several researches show that practising physical and sport activities provide numerous benefits in a cognitive, motor and social level. All these profits have a positive impact in children and their school attendance, also they consolidate values through the physical and sportive activity in Physical Education.
\end{abstract}


Therefore, we understand that it is necessary to facilitate the corporal exercise since from the first stages in our lives, because that moment is essential to assimilate healthy habits that people will maintain along their lives.

After having realised that academic achievements are better in countries and schools where they dedicate more hours to Physical Education, we consider that these data could not be omitted in new laws of education in order to achieve the best educative quality.

Key words: physical-sport activity, benefits, school stage.

\section{Resumo}

Várias investigações demonstram que a prática da atividade físico-desportiva contribui com múltiplos benefícios ao nível cognitivo, motor, psicológico e social. Todos estes ganhos revertêm positivamente nas crianças durante a sua escolarização, para além de consolidar valores através da atividade físico-desportiva, na área da Educação Física. Por este motivo, o acesso e a prática de exercício físico é recomendado desde as primeiras etapas da vida, já que é o momento-chave para forjar hábitos saudáveis que acompanharão as pessoas ao longo da suas vidas.

Logo, verifica-se a existência de melhores resultados académicos em países e escolas onde dedicam mais horas letivas à disciplina de Educação Física. Achamos, por isso, que estes dados não devem ser esquecidos na altura de se fazer reformas educativas, sendo que o que se deseja é a melhoria da qualidade educativa.

Palavras-chave: atividade físico-desportiva, benefícios, etapa escolar.

\section{Introducción}

En las últimas décadas se han publicado numerosos estudios sobre los beneficios de la actividad física. Todos ellos confieren un "poder especial" al ejercicio físico y concluyen que es un elemento clave para alcanzar el bienestar completo. Este planteamiento es perfectamente válido para los alumnos en edad escolar, pues se considera que los efectos positivos que reporta la actividad físico-deportiva se amplían durante la infancia.

Buscar un tratamiento sistémico y completo que forme a los alumnos en todas las dimensiones es uno de los aspectos de máxima actualidad en el mundo educativo. En este sentido, consideramos que la Educación Física no puede quedarse al margen. Según Manzano (2006), esta materia se apoya en el cuerpo y el movimiento para conseguir el desarrollo integral 
del alumno en sus distintos ámbitos (motor o físico, cognitivo o intelectual, afectivo, social y moral). Así pues, la Educación Física debe configurarse de tal forma que ofrezca diferentes respuestas en función de las necesidades del niño. Tal y como defienden Ramírez, Vinaccia y Suárez (2004), únicamente una perspectiva sistémica bio-psico-social-ambiental del ser humano permite entender que las distintas funciones fisiológicas y cognitivas están relacionadas.

En este artículo nos gustaría dejar constancia de los múltiples beneficios que aporta la Educación Física en el desarrollo de los niños en edad escolar, en relación a las características físicas, saludables, de bienestar y psicológicas. Muchos beneficios en cuanto rendimiento académico, la cognición y la trasmisión de valores se encuentran directamente relacionados con la práctica de ejercicio físico como veremos con posterioridad.

\section{Importancia y beneficios de la actividad física}

Algunos estudios, como el de Ramírez (2004), demuestran que el sedentarismo es un factor clave en la aparición y gravedad de diversas enfermedades crónicas. Según apunta este autor, un $20 \%$ del presupuesto destinado a los organismos y entidades sanitarias se deben a las altas tasas de inactividad física. Además, la falta de actividad física en los niños contribuye a la obesidad, al letargo, al bajo tono muscular, a más períodos de tensión con sus referentes adultos, a desarrollar enfermedades como la diabetes, la hipertensión arterial, la hipercolesterolemia, el asma y otros problemas osteo-articulares (Drobnic, 2013).

Ante esta situación, un nivel adecuado de ejercicio físico en la infancia ayuda a garantizar un desarrollo fisiológico óptimo durante las etapas de crecimiento y a prevenir enfermedades infantiles, consiguiendo así uno de los objetivos principales de la Educación Física marcado por la administración, combatir el sedentarismo, con el fin de procurar un desarrollo fisiológico óptimo (Drobnic 2013).

A pesar de la creencia general, la mayor reducción de la actividad física a lo largo de la vida no se produce en la edad adulta, sino que comienza mucho antes, específicamente en el período prepuberal, que abarca entre los 10 y los 13 años - entre $4^{\circ}$ de EP y $1^{\circ} \mathrm{ESO}$-, en función del sexo y de las características individuales de cada uno. Esta etapa viene marcada por una serie de cambios físicos, sociales, psicológicos, morales y emocionales que provocan variaciones y modificaciones en los hábitos diarios, el estilo de vida, la forma de ocupar el tiempo de ocio, y por supuesto, en el tiempo que emplea en actividades físicas (González y Portolés, 2014). En la misma sintonía Drobnic (2013) apunta que el sedentarismo aumenta en la 
etapa prepuberal debido a que el propio desarrollo del niño tiende a disminuir la actividad motora de forma natural; también influyen factores socioculturales, que se manifiestan a través del desarrollo tecnológico y de la presión y competitividad académica, que en ocasiones motiva a que se sustituyan actividades extraescolares deportivas por otras de contenido puramente académico.

Asimismo, el ejercicio físico es un factor importante para evitar conductas que afectan negativamente a la salud, como son el tabaco, el alcohol y el consumo de drogas (González y Portolés, 2014). Por otro lado, Ramírez et al. (2004) apunta que establecer el hábito del ejercicio físico en edades tempranas -desde la escuela-, contribuye a reducir el riesgo de padecer enfermedades mentales a corto y largo plazo.

Los resultados de cuantiosas investigaciones han sacado a la luz datos que revelan la relación directa entre personas con síntomas depresivos y ansiedad y la falta de actividad física. Así, un estudio de Long y Stavel (1995), citado por Ramírez et al. (2004), demuestra que llevar a cabo una actividad aeróbica durante 30 minutos o más tiene fuertes efectos positivos en la reducción de la ansiedad, por lo que podemos afirmar que la práctica de ejercicio físico favorece la construcción de una autoestima más sólida, genera una auto-imagen positiva y mejora también la calidad de vida de las personas. Esta idea es compartida por diferentes autores Morilla (2001) y por Cocke (2002), citado por Scheuer y Mitchell (2003), Drobnic (2013) y Manzano (2006).

A nivel físico los beneficios que aporta la Educación Física son múltiples. González (2011) apunta que tras la práctica de ejercicio físico en el aparato motor se estimula la formación de las células óseas para definir los huesos, se fortalecen los cartílagos y se mejora el riego sanguíneo, además de mejorar la movilidad y elasticidad de los ligamentos articulares; en cuanto al aparato respiratorio, se consigue una mejoría en los músculos que intervienen en la respiración y en el volumen respiratorio; en el sistema cardiovascular se agranda la cavidad cardiaca y se fortalecen las paredes del corazón, disminuyendo así la frecuencia cardiaca y la tensión arterial; el sistema endocrino estimula la secreción de hormonas relacionadas con el crecimiento y el rendimiento físico y mejora la capacidad de destrucción de los productos de desecho de metabolismo; referente al sistema nervioso, también los beneficios que aporta la actividad física son abundantes, pero se describirán minuciosamente más adelante cuando se trate la cognición. 
No hay que olvidar que la práctica de ejercicio físico mejora el desarrollo de la coordinación en general y favorece por tanto el crecimiento armónico del individuo.

Así pues, ser físicamente activo también ayuda a reducir las conductas auto-destructivas y antisociales en la población joven (Ramírez et al., 2004). Por lo tanto, podemos decir que a través de la Educación Física se promueven igualmente beneficios psicológicos en los niños en dos direcciones: evitando enfermedades y problemas mentales - ansiedad, depresión o trastornos de la conducta alimentaria- y favoreciendo la construcción de una auto-imagen y autoestima adecuadas.

\section{Educación Física y rendimiento académico}

Uno de los objetivos básicos del sistema educativo es lograr que los alumnos alcancen el mayor rendimiento académico posible. Para ello se han realizado múltiples propuestas, especialmente durante los últimos años, y la Educación Física y el campo del ejercicio físico no se han quedado atrás.

En primer lugar, es necesario definir qué entendemos por rendimiento académico. Según González y Portolés (2014: 53) puede definirse como: "la productividad del sujeto, el producto final de la aplicación de su esfuerzo, matizado por sus actividades, rasgos y la percepción más o menos correcta de los cometidos", es decir, "el producto que rinde o da el alumnado en el ámbito de los centros de enseñanza, y que normalmente se expresa a través de las calificaciones escolares".

La inmensa mayoría de las investigaciones han demostrado que la actividad física tiene consecuencias positivas en el rendimiento académico, pero con resultados variables, según los parámetros de ejercicio físico y académicos que se hayan medido. A continuación se detallarán algunas, realizando una división entre las que demuestran que la actividad física aporta un beneficio directo al rendimiento académico, y por otro lado, aquellas que concluyen que las mejoras escolares sean indirectas, como consecuencia de los beneficios cognitivos, psicológicos, físicos...

Dentro del primer grupo, destacan dos estudios mencionados por Singh et al. (2012) en su revisión bibliográfica. A cada una de las investigaciones recopiladas les han atribuido un porcentaje de validez en función de las pruebas medidas y la metodología llevada a cabo. Los dos estudios con mejor aplicación metodológica avalan una relación directa y positiva entre el ejercicio físico y el rendimiento académico. El estudio elaborado por Nelson y Gordon-Larsen 
(2006), con un 70\% de puntuación de calidad, comparó las notas de los niños que realizaban actividad física extraescolar con aquellos que no hacían ningún tipo de ejercicio físico fuera del colegio, revelando una correspondencia positiva entre actividad física y rendimiento académico. Mientras que, con un 75\% de puntuación de calidad, la investigación de Donnelly et al. (2009) se basaba en impartir 90 minutos más de clase de Educación Física a la semana a alumnos de entre 9 y 11 años. Los resultados demostraron de nuevo una mejora en las disciplinas académicas en el grupo de niños que había incrementado la duración de las clases de Educación Física. Por tanto, los autores establecieron una correlación directa entre el rendimiento académico y los niños que estaban físicamente más activos.

Del mismo modo, destacan otros estudios como los de Sallis et al. (1999), que comprobaron mejoras en habilidades del lenguaje y en destrezas de lectura, tras añadir 30 minutos de actividad física semanales con 754 niños de 9,4 años de media de edad.

Asimismo, tanto Castelli et al. (2009) citado por Drobnic (2013), como EstebanCornejo et al. (2014), demostraron que los alumnos con mayor capacidad cardiorrespiratoria y una habilidad motora más desarrollada, tanto por separado como en conjunto, tienen mejores resultados académicos en Matemáticas y Lengua.

Ramírez et al. (2004) cita múltiples investigaciones, como la de Dwyer et al. (2001), Linder (2002) o la de Tremblay et al. (2000), que prueban que la dedicación de un tiempo sustancial a actividades físico-deportivas en los colegios provoca beneficios en el rendimiento académico de los niños. En otro estudio recogido por Ramírez et al. (2004), Mitchell (1994) concluye que existe una relación entre los logros académicos de los niños y las habilidades motoras procedentes de una activación física constante. A su vez, Geron (1996), demuestra una correlación positiva entre la coordinación a nivel físico y motor de los niños con buenos resultados académicos, especialmente en Matemáticas y lectura.

Otros dos estudios realizados por Shephard (1984), recogidos por Ramírez et al. (2004), han señalado claros beneficios de la actividad física en el rendimiento escolar. En el primero de ellos se compararon dos escuelas durante 9 años; mientras una proponía entre 1 y 2 horas diarias de Educación Física, la otra se centraba especialmente en las asignaturas más académicas. Después del período de estudio, los alumnos con más horas de Educación Física mostraron mejor salud, actitud, disciplina, entusiasmo, y sobre todo, rendimiento académico. En el segundo estudio se recoge la mejora de una escuela que se encontraba un $25 \%$ por debajo del 
rendimiento académico respecto al resto de colegios del mismo distrito. Tras aplicar un plan de estudios basado en diferentes artes -danza diaria, expresión corporal, música, drama y artes visuales-durante 6 años, pasaron a estar un 5\% por encima de la media de rendimiento escolar de los colegios de su ámbito de referencia.

Ardoy et al. (2014) llevaron a cabo un estudio con alumnos a los que se les amplió la intensidad y las horas de Educación Física, reduciendo así el número de horas en el aula de otras asignaturas, y con alumnos que mantenían el mismo número de clases de Educación Física. Los resultados demostraron un mejor rendimiento académico del primer grupo de alumnos, por lo que una vez más se demuestra que la práctica de actividad física mejora el rendimiento académico.

Otro estudio de Ericsson (2008) pone de manifiesto que tras aumentar la frecuencia de las clases de Educación Física, de dos veces por semana a una al día, los alumnos mostraron un mayor rendimiento escolar. Por otro lado, Ardoy et al. (2014) nombra un estudio llevado a cabo por Devis et al. (2011) con niños con sobrepeso, los cuales mejoraron su respuesta cerebral, su activación cognitiva y su rendimiento matemático.

Destaca también el estudio de Scheuer et al. (2003) realizado en 2002 por el Departamento de Educación de California, en el que participaron más de 900.000 alumnos de entre 10 y 15 años. Después de medir la actividad física y el rendimiento escolar de los alumnos, los datos reflejaron que aquellos con niveles más altos de ejercicio físico tenían los mejores rendimientos académicos, especialmente en Matemáticas y en lectura, tal y como demostraban los estudios nombrados anteriormente. A las mismas conclusiones llegaron Dwyer et al. (2001), citado por Scheuer (2003), tras realizar una investigación con 7.961 alumnos australianos, de entre 7 y 15 años.

Esteban-Cornejo et al. (2014) demuestran que la mejora de la capacidad cardiorrespiratoria y de la habilidad motora que se desarrolla con la práctica de ejercicio físico son los factores que más beneficios aportan al rendimiento académico. La ganancia de la capacidad cardiorrespiratoria se debe a que se forman vasos sanguíneos a partir de otros ya existentes, aumentando así la circulación de la sangre; también, la actividad física aeróbica incrementa los valores de los factores neurotróficos, los cuales promueven la supervivencia de las neuronas y, por último, la capacidad cardiorrespiratoria está relacionada con otros elementos 
cognitivos que mejoran y ayudan al control de los impulsos. Por lo tanto, todos estos fenómenos afectan positivamente al rendimiento académico.

Además de mejorar la coordinación óculo-manual y la motricidad fina mediante la práctica de la actividad física, la mayoría de los niños que la realizan de forma habitual tienen un mejor comportamiento, por lo que repercute en el rendimiento académico y en los procesos de aprendizaje (Cocke, 2002; Dwyer et al., 1983; Shephard, 1997; Tremblay, Inman y Willms, 2000; citados por Ramírez et al., 2004).

Del mismo modo, la actividad motora sirve como fuente de canalización de la energía de los más pequeños y también de diversión (Sánchez y Daniel, 2004), consiguiendo así mayores niveles de atención cuando los alumnos vuelven a clase a recibir los conocimientos (Linder, 1999; citado por Ramírez et al., 2004, y Scheuer et al., 2003). En esta dirección, Ardoy et al. (2014) exponen que al activar a los alumnos en las clases de Educación Física y situarlos fuera del aula, donde pasan gran parte de la jornada escolar, se favorece la relajación de estos.

A mayores de los estudios nombrados, existe una amplia bibliografía que reafirma los beneficios de la actividad física en el rendimiento académico, como los planteados por Kuhlman y Schweinhart (1999), Dwyer, Sallis, Blizzard, Lazarus y Dean (2001), Tremblay et al. (2000), Linder (2002), recopilados por Scheuer et al. (2003); los de Sibley y Etnier (2003), Hillman et al. (2008), Tomporowski et al. (2008), Trudeau y Shephard (2008), Fox et al. (2010), Rasberry et al. (2011), recogidos por Ardoy et al. (2014); los de Coe et al. (2006), Kwak et al. (2009), citados por Ardoy et al. (2014) o el de Esteban-Cornejo et al. (2014).

\section{Educación Física y cognición}

La cognición se refiere "a la facultad de procesar información a partir de la percepción, el conocimiento adquirido (experiencia) y características subjetivas que permiten valorar la información" (Drobnic 2013:10), a la "acción y efecto de conocer" (RAE). Podríamos decir entonces que la cognición es aquel proceso en el que se ponen en marcha diferentes mecanismos con el fin de crear conocimientos nuevos.

Toda la bibliografía consultada demuestra que la actividad física tiene beneficios en la actividad cerebral. Tal y como expone Drobnic (2013), la infancia y la adolescencia son etapas claves para el desarrollo del cerebro, pues este órgano es muy dinámico y en estas fases presenta una mayor capacidad de modificación ante distintos estímulos. Además, durante la etapa de crecimiento el cerebro se modifica con el fin de adquirir y madurar funciones, aprender y 
desarrollarse. Por tanto, toda actividad física que realiza un niño -correr, saltar o jugar- implica la utilización de la energía cerebral y tiene beneficios en el desarrollo y la configuración final del cerebro.

Diferentes estudios realizados en animales y humanos, recogidos por Drobnic (2013), demuestran que el ejercicio físico origina cambios funcionales y estructurales en el sistema nervioso, y en el cerebro en particular. Estas modificaciones positivas para la cognición vienen marcadas por la liberación de un gran número de sustancias como respuesta al ejercicio físico. En concreto se pueden destacar tres grupos de componentes que mejoran a partir de la práctica deportiva (Drobnic, 2013; Ardoy et al., 2014): el aumento de la concentración de neurotransmisores, que fomenta el desarrollo de la memoria; el crecimiento de factores neurotróficos - proteínas que favorecen la supervivencia de las neuronas-, y el incremento del número de neuronas, capilares sanguíneos y conexiones neuronales. Al mismo tiempo, se desarrollan diferentes partes y tejidos cerebrales, como el hipocampo, una estructura del cerebro que tiene un peso importante en la memoria y el manejo del espacio. Todos estos factores son esenciales en procesos cognitivos relacionados con el aprendizaje y la memoria.

Otras evidencias de las aportaciones del ejercicio físico a la cognición se reflejan en estudios recogidos por Ramírez et al. (2004), como el de la Universidad de Illinois (1999), que pone de manifiesto que a mayor actividad aeróbica hay menor degeneración neuronal; o el de Neeper, Pinilla, Choi y Cotman (1996), quienes probaron que el ejercicio aeróbico activaba diversos componentes celulares y moleculares del cerebro.

Respecto a las mejoras en la memoria, Drobnic (2013) cita estudios que demuestran que una sesión de actividad física de 40 minutos mejora la memoria verbal a corto y largo plazo.

Tanto Thayer, Newman y McClain (1994), nombrado por Ramírez et al. (2004); como Ericsson (2008), citado por Ardoy et al. (2014), y Esteban-Cornejo et al. (2014), demostraron una relación positiva entre la actividad física y la secreción del factor neurotrófico cerebral, que produce beneficios en la supervivencia de las neuronas y protege al cerebro frente a la isquemia y otras dificultades cognitivas. Asimismo se producen ciertas hormonas que revitalizan las neuronas (Polanco et al., 2013).

En este mismo sentido, un estudio de Winter, Breitenstein, Mooren, Voelker et al. (2007), citado por Drobnic (2013), comprobó que al dar dos carreras de 3 minutos a una intensidad alta, aceleraba el aprendizaje verbal y ayudaba en la retención de vocabulario a largo 
plazo. Para llegar a esta conclusión, observaron una correlación positiva entre la concentración de factores neurotróficos después del ejercicio físico con la mejora del aprendizaje y la memoria verbal. Igualmente, se ha comprobado como este tipo de sustancias producidas por la actividad física originan mejoras cognitivas en ancianos y personas con problemas de memoria. A su vez, un estudio de la Duke University de Carolina del Norte (2003), recogido por Ramírez et al. (2004), demuestra una notable mejoría en la memoria tras un programa de ejercicio físico durante 4 meses.

Por otro lado, destaca el estudio de un grupo de investigadores japoneses (BrainWork, 2002), citado por Ramírez et al. (2014), pues tras realizar la investigación con jóvenes a los que se sometieron a entrenamientos basados en carreras moderadas durante treinta minutos, tres veces a la semana, a lo largo de tres meses, mejoraron su atención, el control inhibitorio y la memoria de trabajo como consecuencia de una mejoría en la función del lóbulo frontal del cerebro. Conviene destacar que se percibió que estos progresos cognitivos comenzaban a bajar si se detenía la práctica deportiva. También Ericsson (2008), nombrado por Ardoy et al. (2014), observó que el hecho de incrementar las sesiones de Educación Física de dos a cinco clases semanales, tenía un efecto positivo en la atención.

Además de beneficios a largo plazo también se consiguen mejoras en la atención tras realizar ejercicio físico. Gallota et al. (2012) demostraron que con la actividad física se desencadena un proceso de mejora de la memoria, que comienza con una activación del sistema neuronal del cerebro, se incrementa el nivel de sangre que fluye por sus estructuras, origina una excitación del cerebelo y de la corteza frontal, mejorando así las funciones cerebrales y, por último, aumentando la concentración de las hormonas.

Igualmente, diferentes estudios evidencian las mejoras en una de las partes más importantes del cerebro: el hipocampo. Pereira et al. (2007), señalados por Drobnic (2013), comprobaron que la práctica de ejercicio cardiovascular durante tres meses derivaba en un aumento del volumen sanguíneo en el hipocampo, y a su vez, en una mejora de tareas cognitivas relacionadas con la memoria. Esta misma conclusión es compartida por EstebanCornejo et al. (2014) y Ardoy et al. (2014), quienes afirman, como ya se ha apuntado, que la capacidad cardiorrespiratoria desarrollada con el ejercicio físico induce al incremento del volumen sanguíneo. 
Del mismo modo, el estudio de Erickson et al. (2009) y el de Chaddock, Pontifex, Hillman y Kramer (2011), ambos recopilados por Drobnic (2013), evidenciaron una asociación directa entre el nivel de ejercicio físico y el volumen del hipocampo y los ganglios basales agrupaciones de neuronas que regulan el control y el aprendizaje motor- en niños de entre 9 y 10 años. Sincrónicamente demostraron una relación directa entre el volumen de esta área del cerebro y el rendimiento en tareas que requerían memoria visual y funciones cognitivas de control y ejecución. Estas mismas ideas son compartidas por Polanco et al. (2013) y Ardoy et al. (2014). Por lo tanto, todos estos estudios demuestran que a través de la actividad física se produce una mejora en las funciones y estructuras cognitivas que desencadenan diferentes beneficios a nivel cognitivo, entre otros: en la memoria, la atención, el manejo del espacio y el procesamiento de la información; en las neuronas -aumentando en número y favoreciendo su supervivencia-; en la conservación de la función cognitiva y sensorial del cerebro y en un papel neuropreventivo ante enfermedades neurodegenerativas. Estas mejoras se producen gracias a que el desarrollo cognitivo surge simultáneamente a la habilidad motora.

De esta forma, los resultados mostrados avalan las mejoras cognitivas ocasionadas por la actividad física. Pero existe una doble lectura: no se puede olvidar que la capacidad de adaptabilidad del sistema nervioso, que también se conoce como neuroplasticidad, disminuye con la edad. Por este motivo, es importante que los niños de primaria afiancen hábitos de ejercicio físico, con el fin de procurarse un buen desarrollo cognitivo.

Aun así, teniendo evidencias claras de que la actividad físico-deportiva tiene numerosos beneficios en la cognición y en las estructuras cerebrales, es necesario seguir investigando, ya que la relación entre el ejercicio físico y la cognición es realmente compleja, pues dependiendo del tipo de ejercicio y de la duración de este, se mejora más un aspecto cognitivo u otro (Drobnic, 2013).

Hasta la fecha, la investigación de Esteban-Cornejo et al. (2014) es de las pocas que se han centrado en distinguir los diferentes componentes desarrollados a través del ejercicio físico y cuáles son los beneficios cognitivos que aporta cada uno de ellos; los resultados con niños en edad escolar muestran que la capacidad cardiorrespiratoria mantiene una relación positiva con el aumento del control inhibitorio, la memoria de trabajo y la flexibilidad cognitiva, mientras que la habilidad motora favorece el desarrollo de estas mismas propiedades y de la atención. 
Continuando con esta línea de investigación, hay publicaciones que se centran en conocer el aumento de las sesiones de Educación Física necesario para la obtención de estos beneficios. Por ejemplo, Ardoy et al. (2014) compararon un grupo control con otros dos: en uno simplemente se aumentó el número de sesiones, mientras que en el otro se incrementó la intensidad, además del volumen. Los resultados demostraron que en este último grupo fue en el que se encontraron sustanciales beneficios cognitivos, mientras que en el grupo que simplemente se habían añadidos algunas sesiones de Educación Física, las mejorías eran muy leves. Estos resultados coinciden con los obtenidos en diferentes estudios, como los de Dwyer et al. (1996), Sallis et al. (1999), Ahamed et al. (2007) o Ericsson (2008), citados por Ardoy et al. (2014).

Además de los múltiples estudios citados, existen muchos más que reafirman los beneficios anteriormente expuestos, como Rosenbaum, Carlson y Gilmore (2001), Sibley y Etnier (2003), Hillman, Erikson y Kramer (2005), Reed, Einstein, Hahn, Hooker, Gross y Kravitz (2010), citados por Ardoy et al. (2014); o como los de Shephard (1997), Cocke (2002), Tremblay, Inman y Willms (2000), nombrados por Scheuer y Mitchell (2003); o los de Linder (1999), recogidos por González et al. (2014).

\section{Educación Física y valores}

La importancia de la trasmisión de valores a través de la educación física se explica fundamentalmente por tres ideas: la educación integral del ser humano, las condiciones favorables de la actividad física para la trasmisión de valores y la importancia que ha adquirido el deporte en nuestros días.

El ser humano es considerado una entidad global que abarca tres dimensiones: biológica, psicológica y social. Esto quiere decir que ante cualquier tipo de actividad física se ponen en funcionamiento tres áreas: biomotriz, psicomotriz y sociomotriz. Desde este punto de vista, diremos que cualquier ejercicio físico pone en marcha todas ellas, potenciando también las emociones y las relaciones sociales (Gutiérrez, 2004).

\subsection{Valor: concepto y características}

Con frecuencia asociamos los valores a algo que hemos aceptado de una manera relativamente voluntaria y que nos marcan y caracterizan en nuestro comportamiento. Pero, ¿qué es un valor? Debemos tener en cuenta que es un término polisémico, por tanto, un valor es una creencia 
duradera -no se modifica fácilmente- que además se forma especialmente en la infancia a través de los procesos de socialización.

Los valores tienen un carácter sociológico, por lo que dependen de la sociedad en la que se viva y de las interacciones con la gente que nos rodea.

\subsection{La naturaleza social del deporte y la actividad física}

A pesar que el deporte tiene lugar a partir de la capacidad motriz del ser humano, se trata de un producto, una construcción social de los seres humanos.

El deporte y el juego, que son contenidos de las clases de Educación Física, tienen un enorme potencial socializador, ya que ayudan a los individuos a interiorizar normas y valores sociales (Martos y del Castillo, 2009).

La función socializadora del deporte es tan relevante que en la "Declaración de Niza sobre el deporte", celebrada en el año 2000, puso de manifiesto la importancia social y educativa: "El deporte es una actividad humana basada en unos valores sociales, educativos y culturales esenciales. Es factor de inserción, de participación en la vida social, de tolerancia, de aceptación de las diferencias y de respeto de las normas".

\subsection{Agentes sociales que influyen en la trasmisión de valores a través del deporte}

Los agentes sociales o socializadores son aquellas personas, grupos u organizaciones que influyen en la conformación de la personalidad de un individuo, desde que nace hasta que muere.

La socialización en el deporte se lleva a cabo a través de varios agentes principales: la familia, el profesorado y técnicos deportivos, el centro docente, los amigos y compañeros y los medios de comunicación (Águila, 2013). No obstante, existen otros con un enorme impacto socializador, como las organizaciones deportivas -clubes, federaciones, entrenadores...-. Todos estos agentes son verdaderamente influyentes, máxime cuando nos referimos a niños. Podemos decir que el profesorado es uno de los factores más determinantes en la trasmisión de valores en los centros educativos, por razones obvias, pues el niño pasa mucho tiempo en la escuela. El maestro de Educación Física será uno de los responsables de que el niño haga un buen o mal uso de la actividad físico-deportiva y de la transferencia de valores y hábitos deportivos a otros campos de su vida. 
Algunos autores, como Carranza y Mora (2003), destacan que ciertos aspectos personales como la edad, el género o la experiencia profesional de los profesores, son factores significativos en la trasmisión de valores. Del mismo modo, mantienen que existen ciertos aspectos del estilo docente que tienen también gran influencia, como es la relación con el grupo, el conseguir o no una relación empática, la forma de dirigirse, el tono de voz, la mirada, el contacto físico, el clima que se respire en la sesión...

Si nos centramos en el deporte, la materia de Educación Física adquiere mucha importancia en la educación en valores, pues será en esta asignatura donde muchos niños tengan su primer contacto con las normas, los valores o los hábitos deportivos a través de juegos que irán afianzando y completando progresivamente a lo largo de su etapa educativa (Martos y del Castillo, 2009).

Atendiendo a esta visión, cabe destacar un reciente proyecto realizado en diferentes centro educativos - dentro de la asignatura de Educación Física- de cinco ciudades españolas Salamanca, Guadalajara, Valencia, Badalona y Madrid-, basado en el fomento del deporte y los valores que se trasmiten, "Sport\&Trops". El principal objetivo es acercar ciertos deportes, hábitos saludables, afianzar contenidos de seguridad vial y, especialmente, valores inherentes al deporte a los alumnos a través de deportistas que han tenido o tienen vinculación con la élite.

\section{4 ¿Qué valores se trasmiten a través del deporte?}

Los diferentes valores universales que se pueden trasmitir a través del deporte educativo ${ }^{1}$ según Puig (Cit. en Carranza y Mora, 2003), son aplicables a situaciones interpersonales variadas justicia, libertad, solidaridad y tolerancia-, tienen un contenido útil para enjuiciar-benevolencia, cooperación, respeto, amor y amistad-e influyen en cualquier relación humana -capacidad crítica y autocrítica, autonomía, responsabilidad y voluntad de valor-.

UNICEF (2004:12) muestra una serie de valores que se aprenden a través del deporte son: valores saludables -autoaceptación y cuidado del cuerpo, higiene, calidad de vida, alimentación, cuidado y respeto del nivel evolutivo-, utilitarios -espíritu lúdico, esfuerzo, perseverancia, tenacidad, aprovechamiento del tiempo, autorregulación, motivación, autoconcepto y autocontrol-, morales -respeto y aceptación de las normas, cooperación, tolerancia, cordialidad, compañerismo, honestidad, responsabilidad, generosidad, sentido de

\footnotetext{
1 "Aquel que permite el desarrollo de las aptitudes motrices y psicomotrices, en relación con los aspectos afectivos, cognitivos y sociales de la personalidad del individuo" Le Boulch (Cit. en Martos y del Castillo, 2009:12)
} 
pertinencia, empatía, autoestima, espíritu crítico y constructivo, aceptación de la diferencia, paz, justicia social, solidaridad y respeto al medio ambiente-.

Por último, cabe resaltar uno de los valores fundamentales de cualquier deporte y que en definitiva engloba a la mayor parte de ellos, el juego limpio o "fair play", que implica el reconocimiento y respeto por las reglas de juego, el establecimiento de relaciones correctas con el adversario, el mantenimiento de igualdad de oportunidades y de condiciones para todos, el rechazo de la victoria a cualquier precio, una actitud digna tanto en la victoria como en la derrota y el compromiso real de dar de sí todo lo posible.

Como afirma González (2001), la deportividad está rodeada de honestidad, honradez, hermandad, humildad, honor y humanidad, el resumen del conjunto de valores que se trasmiten a través del deporte.

\section{Conclusiones}

Después de leer múltiples estudios referidos al tema objeto de estudio, se advierte que la Educación Física es una de las vías más asequibles para fomentar la práctica de actividad físicodeportiva en la niñez. En la etapa de educación primaria los beneficios cognitivos derivados de esta práctica tienen mayor énfasis, debido a la gran plasticidad del cerebro y a que coincide con el momento en que se están conformando las diferentes conexiones neuronales y estructuras cognitivas. A ello hay que añadir que la infancia es el momento más oportuno para establecer hábitos saludables y es cuando se asimilan la mayoría de los valores.

Tras demostrar que el ejercicio físico tiene efectos positivos en el rendimiento académico, mejorando especialmente las destrezas en la asignatura de Matemáticas y en la lectura, y también que tras su práctica los niños están más activos, presentan mejor comportamiento, mayor autoestima y autoconcepto y evitan el aburrimiento, nos cuestionamos si no habría que ampliar las horas lectivas de la materia de Educación Física, ya que ciertas investigaciones han puesto de manifiesto que el hecho de reducir horas de asignaturas estrictamente académicas para ampliar las de Educación Física no ha derivado en un peor nivel académico, sino todo lo contrario, ha originado una mejora en el rendimiento escolar.

Teniendo en cuenta los resultados de los distintos estudios como evidencias científicas, es necesario ser críticos con los criterios que se siguen a la hora de modificar las leyes educativas y eliminar parte de las horas y del currículo de Educación Física en las escuelas. 
En la actualidad, tanto la Educación Física como otras actividades motrices, por ejemplo el juego motor, están siendo reducidas o eliminadas del día a día de los niños, repercutiendo negativamente en su rendimiento académico y en su salud, tal como ha quedado demostrado. Según Drobnic (2013), en numerosas ocasiones los padres no apuntan a sus hijos a actividades extraescolares relacionadas con la actividad motora porque prefieren que acudan a clases de refuerzo o apoyo, con el fin de mejorar su rendimiento académico, logrando en muchos casos el efecto contrario.

España es el país europeo que menos tiempo dedica a la Educación Física en el currículo oficial, junto con Malta y Turquía, llegando tan solo a un 3-4\% del tiempo total en la etapa de educación secundaria (Ardoy et al., 2014). Comparando estas cifras con los resultados del Informe PISA (2012) publicados por la OECD (2013), llama la atención que tanto Francia como Austria, dos de los países que más tiempo (14\%) dedican a la Educación Física en el currículo escolar, están por encima en todos los parámetros de otros que tienen menos horas lectivas de esta materia -España, Turquía y Malta-, y en alguno de ellos presentan una diferencia sustancial, como puede comprobarse en la siguiente tabla.

\begin{tabular}{|c|c|c|c|c|c|}
\hline País & $\begin{array}{c}\text { Puntuación } \\
\text { media en } \\
\text { Matemáticas }\end{array}$ & $\begin{array}{c}\text { Puntuación } \\
\text { media en lectura }\end{array}$ & $\begin{array}{c}\text { Puntuación } \\
\text { media en } \\
\text { Ciencias }\end{array}$ & $\begin{array}{c}\text { Proporción de } \\
\text { estudiantes con bajo } \\
\text { rendimiento }\end{array}$ & $\begin{array}{c}\text { Proporción de } \\
\text { estudiantes con alto } \\
\text { rendimiento }\end{array}$ \\
\hline España & 484 & 488 & 496 & 23,6 & 8,0 \\
\hline Turquía & 448 & 475 & 463 & 42,0 & - \\
\hline Malta & 463 & 442 & 461 & - & 12,9 \\
\hline Francia & 495 & 505 & 499 & 22,4 & 14,3 \\
\hline Austria & 506 & 490 & 506 & 18,7 & \\
\hline
\end{tabular}

Tabla 1. Resumen de resultados PISA 2012 en Matemáticas, Lectura y Ciencias. (OECD, 2013).

Si se tiene en cuenta la proporción de estudiantes con bajo rendimiento, los porcentajes son relativamente parecidos entre los tres países -España, Francia y Austria-, aunque siempre obtienen mejores resultados los países con mayor peso de la Educación Física en el currículo. Las diferencias respecto a la proporción de alumnos con alto rendimiento son mucho mayores:

\footnotetext{
${ }^{2}$ Los datos de Malta son del Informe PISA 2009 ya que no participó en la edición del 2012.
} 
en España solo hay un 8,0\%, mientras que Francia tiene un casi un 5\% más y Austria algo más de un $14 \%$.

Somos conscientes de que el rendimiento académico depende de múltiples factores, pero, curiosamente, los países en los que se dedica menos horas a la Educación Física obtienen una puntuación media inferior en el informe PISA en Matemáticas, Ciencias y lectura, un mayor número de estudiantes con bajo rendimiento y menos alumnos con alto rendimiento.

Desde este punto de vista, no se entiende que en las últimas reformas educativas españolas se haya reducido el número de horas lectivas de esta asignatura. Efectivamente, en la ley actual, "Ley Orgánica para la mejora de la calidad educativa", lejos de considerarse las evidencias empíricas extraídas de los datos y conclusiones finales de rigurosas investigaciones, se ha continuado reduciendo horas de Educación Física en algún curso, concretamente 1 hora en

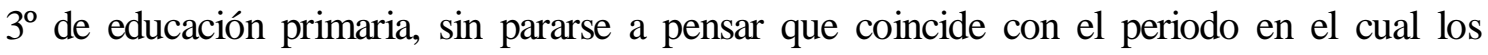
alumnos están adquiriendo y reafirmando hábitos saludables que pueden perdurar a lo largo de su vida.

\section{Referencias bibliográficas}

Águila, C. (2013). Del deporte a la sociedad: sobre valores y desarrollo del ser humano. Jornadas para Formadores de Deportistas en Edad Escolar. Almería: Diputación de Almería.

Ardoy, D. N., Fernández, J. M., Jiménez, D., Castillo, R., Ruiz, J. R. y Ortega, F. B. (2014). A Phisical Education trial improves adolescents' cognitive performance and academic achievement: the EDUFIT study. Scandinavan Journal of Medicine \& Science in Sports, 24, 52-61.

Carranza, M. y Mora, J.M. (2003). Educación física y valores: educando en un mundo complejo. 31 propuestas para los centros escolares. Barcelona: Graó.

Consejo Europeo de Niza. (2000). Declaración de Niza sobre el deporte. Niza: Consejo Europeo de Niza.

Drobnic, F. (Coord.) (2013). La actividad física mejora el aprendizaje y el rendimiento escolar. Los beneficios del ejercicio en la salud integral del niño a nivel físico, mental y en la generación de valores. Cuadernos Faros, 7, 5-29. 
Esteban, I., Tejero, C. M., Martínez, D., Del Campo, J., González, A., Padilla-Moledo, C., Sallis, J. y Veiga, O. L. (2014). Independent and Combined Influence of the Components of Physical Fitness on Academic Performance in Youth. The journal of pediatrics, $165,306-312$.

Gallota, M. C., Guidetti, L., Franciosi, E., Emerenziani, G. P., Bonavolontà, V., y Baldari, C. (2012). Effects of Varying Type of Exertion on Children's Attention Capacity. Medicine \& Science in Sports \& Exercise, 44, 3, 550-555.

González, J. y Portolés, A. (2014). Actividad física extraescolar: Relaciones con la motivación educativa, rendimiento académico y conductas asociadas a la salud. Revista Iberoamericana de Psicología del Ejercicio y el Deporte, 9, 1, 51-65.

González, F. (2001). Educar en el deporte. Educación en valores desde la Educación Física y la animación deportiva. Madrid: Editorial CCS.

Gutiérrez, D. (2007). Modelo de intervención para educar en valores a través del fútbol: una experiencia con entrenadores de la Comunidad de Madrid. (Tesis de maestría. Universidad Politécnica de Madrid). Consulta realizada 10 de diciembre de 2015. http://oa.upm.es/6582/1/DIEGO_GUTIERREZ_DEL_POZO.pdf

Gutiérrez, M. (2004). El valor del deporte en la educación integral del ser humano. Revista de Educación, 335, 105-126.

Hernández, I., Martínez, L. F. y Águila, C. (Coords.). (2008). El deporte escolar en la sociedad contemporánea. Almería: Editorial Universidad de Almería.

Manzano, J. (2006). Educación Física y Desarrollo Integral. Isla de Arriarán, XXVIII, 275-294.

Martos, P., y del Castillo, J. (2009). Fundamentos sociológicos del deporte.

OECD. (2013). Resultados PISA 2012: Lo que los estudiantes saben y pueden hacer Desempeño de los estudiantes en matemáticas, lectura y Ciencias. Publicación OECD, I, PISA.

Polanco, I., Álvarez, M. M. y Nájera, G. (2013). XVI Congreso Internacional sobre Innovaciones en Docencia e Investigación en Ciencias Económico Administrativas. Caracterizar los factores de la actividad física y el deporte, sobre el rendimiento 
académico de los alumnos que cursan la materia de Aspectos Básicos de Contabilidad, Chihuahua: Universidad Autónoma de Chihuahua.

Ramírez, W., Vinaccia, S., y Suárez, G.R. (2004). El impacto de la actividad física y el deporte sobre la salud, la cognición, la socialización y el rendimiento académico: una revisión teórica. Revista de Estudios Sociales, 18, 67-75.

Ruíz, G., y Cabrera, D. (2004). Los valores en el deporte. Revista de Educación, 335, 9-19.

Sánchez, Ch., y Daniel, M.J. (2004). El juego, instrumento educativo, Revista Foro de Educación, 2, 15-17.

Scheuer, L. J., y Mitchell, D. (2003). Does Physical Activity Influence Academic Performance? The new P.E. \& Sports Dimension.

Singh, A., Uijtdewilligen, L., Twisk, W. R., van Mechelen, W. y Chinapaw, J. M. (2012). Physical Activity and Performance at School. American Medical Association, 166, 1, 49-55.

UNICEF. (2004). Deporte para un mundo mejor. Guía para la práctica deportiva. Madrid: UNICEF. 